\title{
OPTIMIZATION OF HYDROSTATIC-MECHANICAL TRANSMISSION CONTROL STRATEGY BY MEANS OF TORQUE CONTROL
}

\author{
M. Sc. Yusheng Xiang*, Dr. Steffen Mutschler, Dipl.-Ing. Norman Brix, Dr. Christine \\ Brach, Prof. Dr. Marcus Geimer
}

Department of system engineering, Bosch Rexroth AG, Glockeraustr. 2, 89275 Elchingen, Germany

* Corresponding author: Tel.: +49 17672361970; E-mail address: Yusheng.xiang@boschrexroth.de

\begin{abstract}
The combination of hydrostatics and mechanical gearboxes cannot only improve system efficiency but also the usability of mobile machines. A possible solution could be a synchronized gearbox with two gears. Compared to the pure hydrostatic drivetrain, mobile machines with such a combination can have a larger drive torque with the first gear and higher efficiency at high speed. However, the calibration effort of this traditional drivetrain system is enormous; moreover, with a flow-based control, the degrees of freedom to achieve optimum shift performance in all conditions are limited. By adopting the primary torque control concept, we propose a novel hydrostatic-mechanical drivetrain control algorithm so that the holistic shift performance is better whilst the calibration effort is dramatically reduced by systematically adjusting only one hyper-parameter. Experienced engineers and machine operators validated the advantages mentioned here by a series of field experiments. Besides synchronized gearboxes, the controller proposed in this paper is also generalized to other kinds of mechanical transmissions.
\end{abstract}

Keywords: primary torque control, hydrostatic-mechanical transmission, control strategy, mobile machine

\section{INTRODUCTION}

The combination of hydrostatic and mechanical gearboxes cannot only improve system efficiency but also the usability of mobile machines. A possible choice could be a synchronized one with two gears. Figure 1 illustrates the hydrostaticmechanical drivetrain solution. Instead of directly connecting a hydraulic motor to the vehicle axle, a synchronized gearbox is installed between them. With such a drivetrain system, mobile machines can have two different drive ranges, such as a torque range and a speed range, with only one hydraulic motor. Due to favourable cost in comparison with multi-motor solutions, hydrostatics with synchronized gearbox dominates the market for medium-sized mobile machines with high speed capability up to 40 $\mathrm{km} / \mathrm{h}$. However, this successful drivetrain solution is rarely mentioned in academia. The main challenge is that current control solutions commonly adopt a flow-based approach resulting in complex control algorithms for the operation of the hydrostat and synchronizer actuators. Although a flow-based hydrostatic-mechanical transmission control strategy is well proven on the market, it still can be further optimized for simplicity resulting in better calibration effort, shift management, and shift process performance. In this paper, we are going to propose an alternative control concept for the drivetrain system shown in Figure 1.

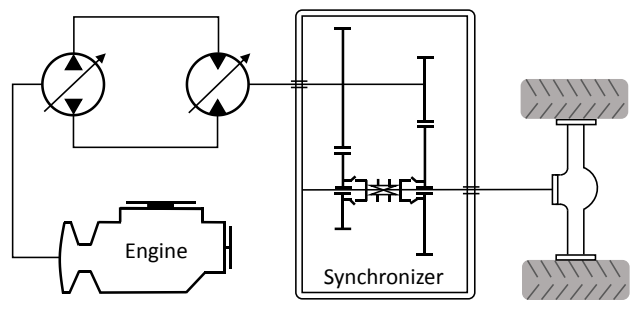

Figure 1: System layout of hydrostatics in combination with a synchronized gearbox

\section{STATE OF THE ART}

Currently, most of the mobile machines are equipped with hydrostatics or hydrodynamics transmissions [1]. Mobile machines with an 
electric motor are typically only used in some special areas, such as a sealed working site.

The basic principle of a transmission with hydrodynamic torque converter on mobile machines is the same as the automatic transmission in passenger cars. Due to its smooth drive characteristic and thus relatively better drive comfort, hydrodynamics is still a preferable solution in North America and Asia [2].

By contrast, hydrostatics can decouple the engine rotational speed from vehicle velocity and torque so that the engine can theoretically work in its best efficient region resulting in a higher holistic efficiency up to $20 \%$ when combine with power split transmission [3, 4]. One major reason for this high consumption gap is the fact that, at low drive speed high engine speeds are needed for the implement hydraulics. If decoupling of both states is not given, excess drive torque from the engine is dissipated by the brake or in the torque converter.

As a consequence of that, torque control became one of the research trends for the hydrostatic drivetrain, which mainly aims at improving the drive comfort of hydrostatic mobile machines, reducing the application effort and introducing open, comprehensible software interfaces known from the automotive industry. At present, we can classify torque control into secondary control and primary control. Figure 2 describes the definition of different control concepts, according to Murrenhoff $[5,6]$.

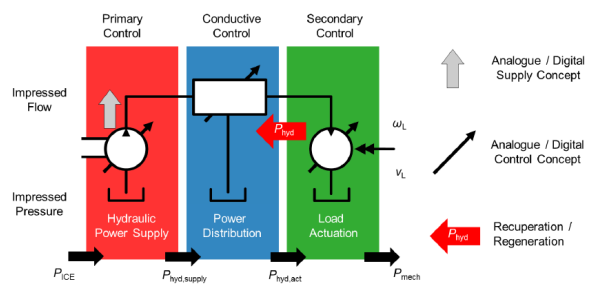

Figure 2: Fundamental structure of a mobile hydraulic system [6]

\subsection{Secondary (torque) control}

The concept of secondary control was brought forward in the 80's [7]. With more than 30 years of development, there are many variants of secondary control concept $[8,9]$. However, the basic idea remains the same: the pressure is set to the desired level whilst the torque is controlled by the swivel angle of the motor.
A series of researchers have implemented secondary control in their applications. Among them, the most notable applications are STEAM for open circuit [10-12] and the concept proposed by Sprengel for closed circuit $[13,14]$. Because the focus point of this paper is drivetrain, a more detailed description of STEAM is out of the scope.

Generally, a naive secondary controller uses the motor to control its drive direction and the torque outputted by hydraulic motor, resulting in a dilemma for mobile machine reversing. On the one hand, the swivel angle of hydraulic motor should be large so that the torque is large while approaching velocity zero. On the other hand, the hydraulic motor's swivel angle must go to zero to change its rotation direction. To solve this problem, Sprengel at Purdue University further developed the secondary drivetrain solution by adding a valve which allows the vehicle to smoothly change its drive direction. However, the system is complicated not only in hardware but also in software, compared to primary torque control.

We believe that secondary control is particularly useful for applications that make lots of reciprocating motion due to its excellent recuperation ability. However, in the case of mobile machines of which typical working process is reversing, secondary concept is still too expensive. As a light of that, we would like to go ahead with primary torque control.

\subsection{Primary torque control}

Primary torque control was first published in 2018 [2]. In contrast to secondary control, the primary torque controller controls the pressure instead of the motor swivel angle by adapting the pump swivel angle.

The advantages of this control algorithm are enormous. First and foremost, standard drivetrain components can be used and thus cost is lowered. Secondly, the swivel angle of the pump is always at a high level entailing a relatively lower energy loss. Thirdly, a constant motor can be used for this drivetrain. Fourthly, the pressure in the circuit is also adapted to the desired value and thus avoids system loss due to too high pressure. Last but not least, no accumulator is needed and therefore there is no risk of stored energy. 


\subsection{Synchronized gearbox for hydrostatic mobile machines and corresponding control algorithm}

The success of this drivetrain mainly lies in the fact that it can shift without stopping the mobile machines [15]. The synchronized gearbox is slightly different from the standard manual gearbox on passenger cars: it does not have a neutral position. That is, either the first gear or the second is engaged. If there is still an active torque exerting on the input shaft during the shifting process, exactly in synchronization process, the destruction of the synchronizer is sure to ensue [16]. As a result of that, tractive effort is diminished to zero in order to shift the gear.

The-state-of-the-art hydrostatic-mechanical drivetrain solution uses a flow-based control concept. The first typical character is that the pressure inside of the closed-circuit is depended on the external load. The second is that the control variable is usually vehicle velocity or a rotational speed regard of vehicle velocity. For the sake of simplicity, we can summarize the control strategy as follows. The drive pedal controls the engine speed and the pump swivel angle resulting in the volume flow supplied by the hydraulic pump. In the meantime, the hydraulic motor is set to a certain angle depending on motor rotational speed. Consequently, the drive pedal determines the vehicle speed individually if the mechanical gear does not change. One disadvantage is that the desired velocity is set to different values with different gears which could cause a vehicle jerk during shifting with constant pedal position. The effect can be compensated with some additional software functionality; however, this leads to much higher calibration effort and more complicated control algorithms.

In the next section, we are going to describe a simple yet efficient control algorithm, which needs only little calibration effort while also has a better shift performance.

\section{SHIFT CONTROL ALGORITHM}

As mentioned above, during the synchronization process, the hydraulic motor should not produce an active torque. Formally, the torque can be calculated as

$$
T_{m}=\frac{V_{g, \max } \cdot \alpha_{m} \cdot \Delta p \cdot \eta_{m e}}{2 \pi}
$$

where $T_{m}$ is the output torque of the hydraulic motor, $\alpha_{m}$ is the swivel angle of hydraulic motor, $\Delta p$ is the actual pressure difference inside of the closed circuit, $\eta_{m e}$ is the mechanical loss coefficient. Here either the swivel angle or the pressure must be zero to enable the shifting process. Based on these two concepts, two control algorithms are proposed: swivel-angle based control algorithm (ABCA) and pressurebased control algorithm (PBCA). We firstly implemented both of them in Simulink. With a plant model in Amesim, the performance and feasibility of both algorithms are virtually validated based on basic requirements.

No matter which algorithm we use, the control scheme is the same, as shown in Figure 3. In order to simplify the complexity of the shift control system, we design the system, which uses 2-DoF-controller to reduce the calibration effort

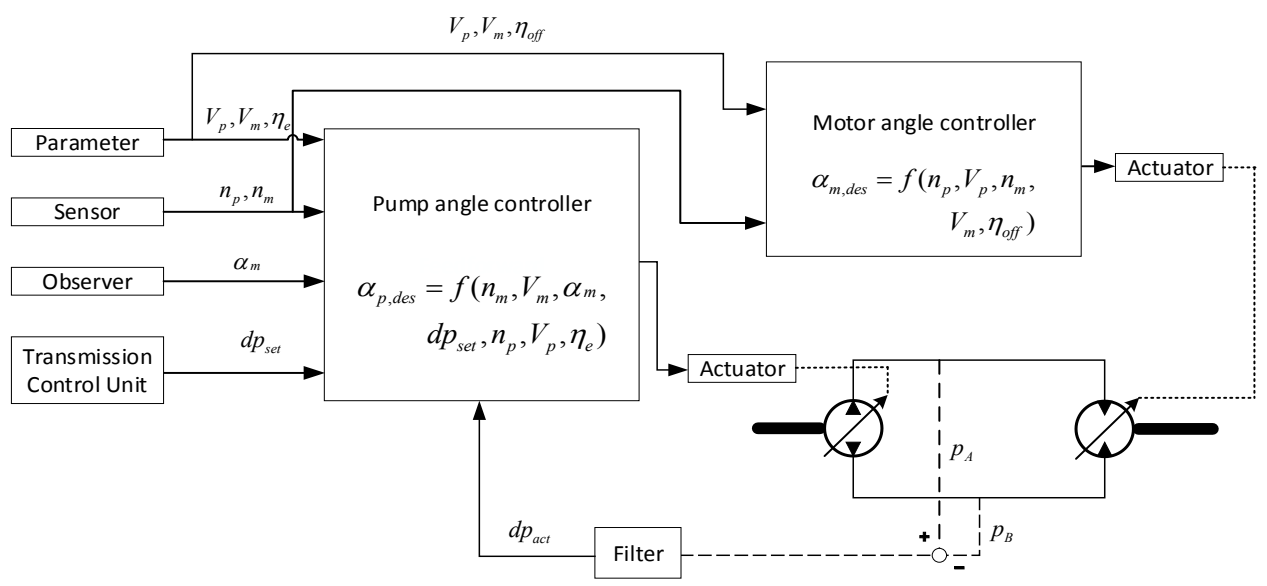

Figure 3: Novel control schema of the hydrostatic-mechanical drivetrain 
having an interface so that engineers can systematically adjust the shifting performance with only one calibration parameter. Concretely, we adjust a time constant parameter in software sensor, which usually is used to estimate the motor angle. The time constant affects the output of observer and thus the compensation function of pump controller.

\subsection{Pressure-based control algorithm}

The basic idea of the pressure-based control algorithm is to adapt the pressure inside of the closed-circuit to zero so that the swivel angle of the motor can keep the same angle as before the shifting. Due to relatively higher dynamics, the shift duration is potentially shorter than the anglebased control algorithm if the pressure-based control algorithm is used.

Since the rotational speed of the hydraulic motor in the first gear is higher than that in the second gear at the same speed, the motor is accelerated during the synchronization process by downshifting. However, the swivel angle of the pump cannot always go up to compensate for the pressure change. Thus, for the downshifting process, a preparation step should be added to the entire shift process. Concretely, the swivel angle of the motor is reduced to a certain low level before synchronization process so that the swivel angle of the pump is also set to a lower level. The drive torque will not diminish since the pressure will correspondingly increase. Figure 4 shows the control flow of shifting process with this algorithm.

\section{Simulation result of $P B C A$}

To study the feasibility of this concept, we built up a co-simulation environment in Simulink and Amesim. The parameters we used are chosen from the Bosch Rexroth A6 motor and A4 pump datasheet. The length of pipe plays no role in this simulation since we use torque control. We shift the gear at vehicle speed of $5 \mathrm{~km} / \mathrm{h}$ since it is the most common downshift speed. As visible in Figure 5, the pressure is reduced to zero and then the synchronization process occurs. In this way, the shift duration is even shorter than 1 second. However, the pressure is quite difficult to maintain at zero during the synchronization since this process is very quick. In this phase, the pump

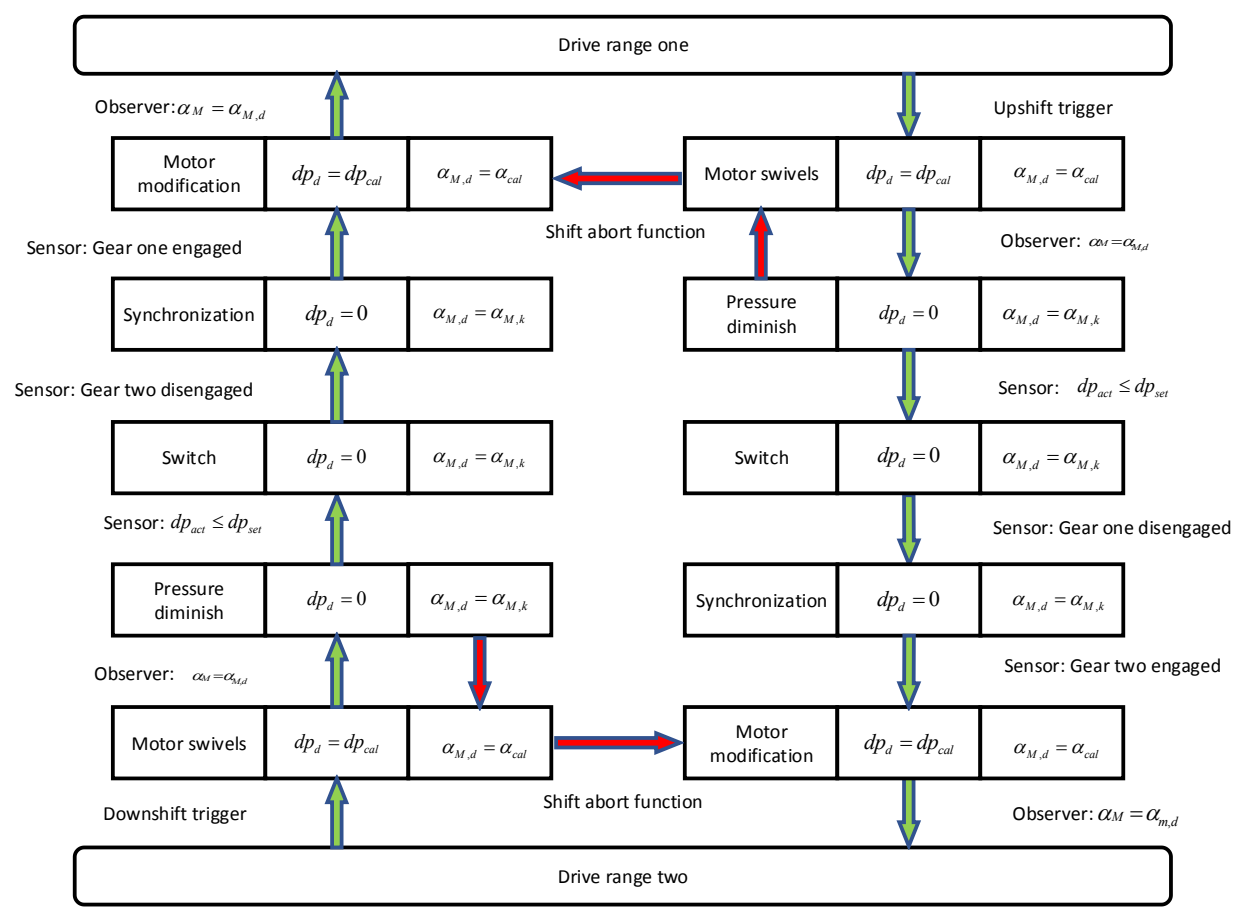

Figure 4: Control flow of Pressure based control algorithm 

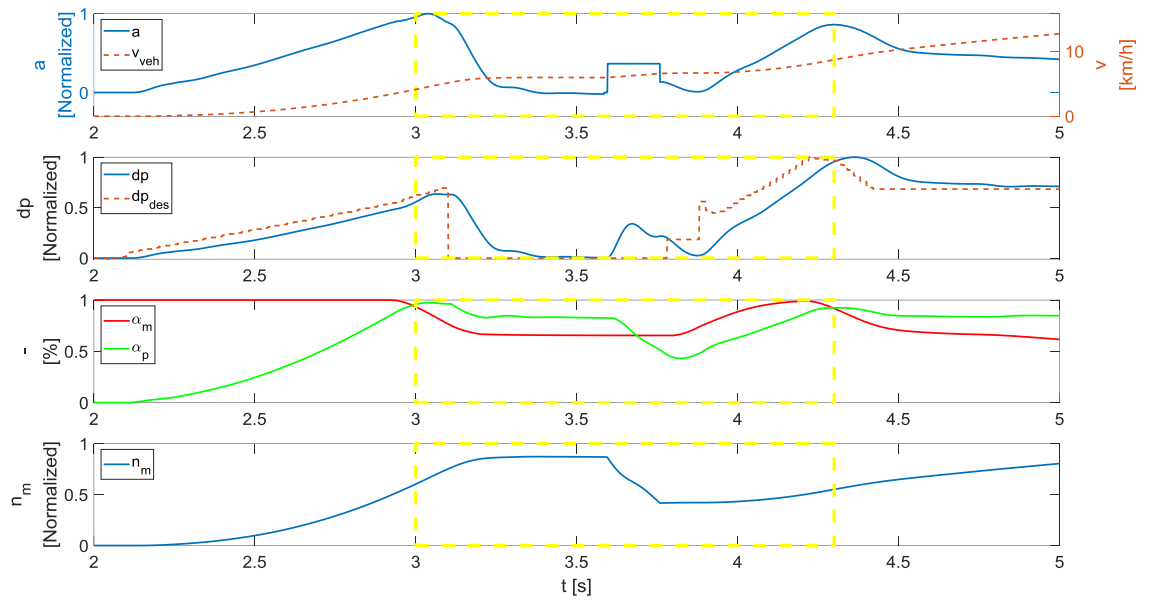

Figure 5: Simulation result of shifting with pressure based control algorithm

swivels back to compensate the effect due to the deceleration of the motor. Nevertheless, a pressure higher than 10 bar occurs, which will certainly damage the synchronizer. Moreover, we did the simulation at the vehicle speed of only $5 \mathrm{~km} / \mathrm{h}$. Shifting at a higher speed with a larger rotational speed difference, the situation will exacerbate. Consequently, pressure-based concept might not be a viable solution or it needs further development. The shift process is demonstrated in the yellow rectangle.

\subsection{Swivel-angle based control algorithm}

By contrast, the swivel-angle-based control algorithm makes the motor swivels to its zero position. Figure 6 demonstrates the flow of this control algorithm.

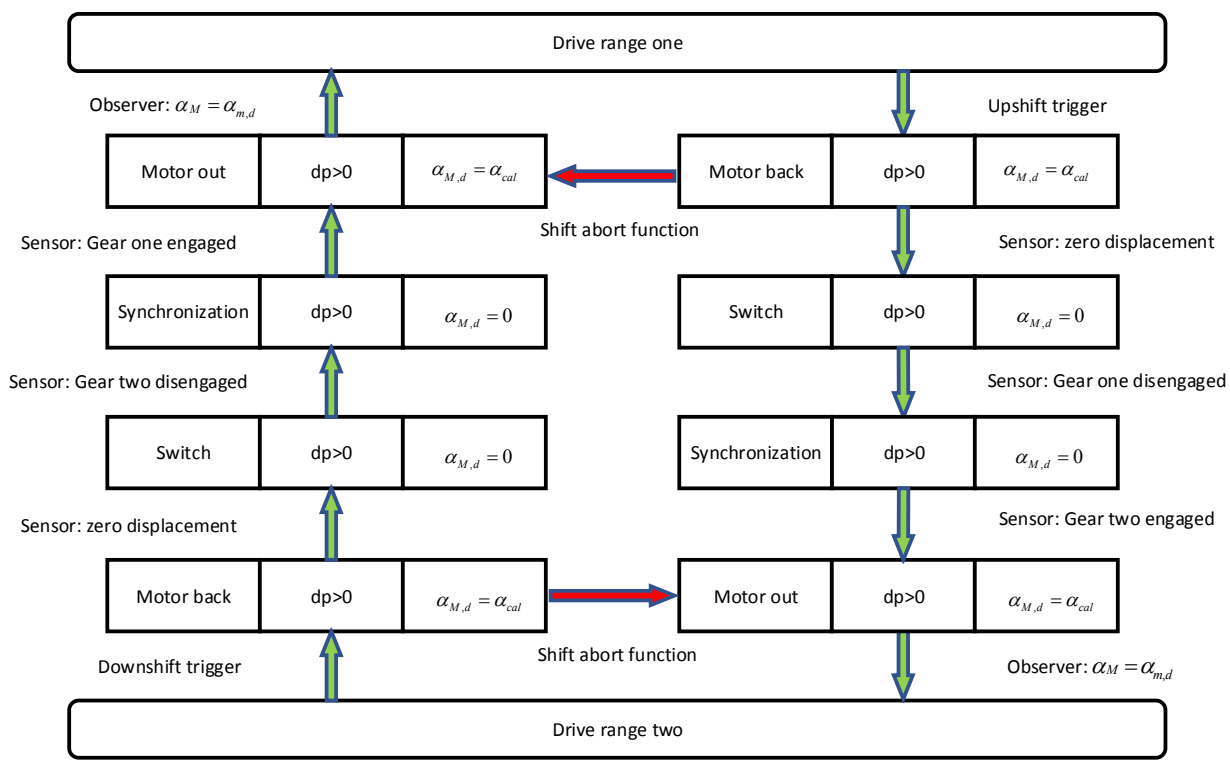

Figure 6: Control flow of angle based control algorithm 


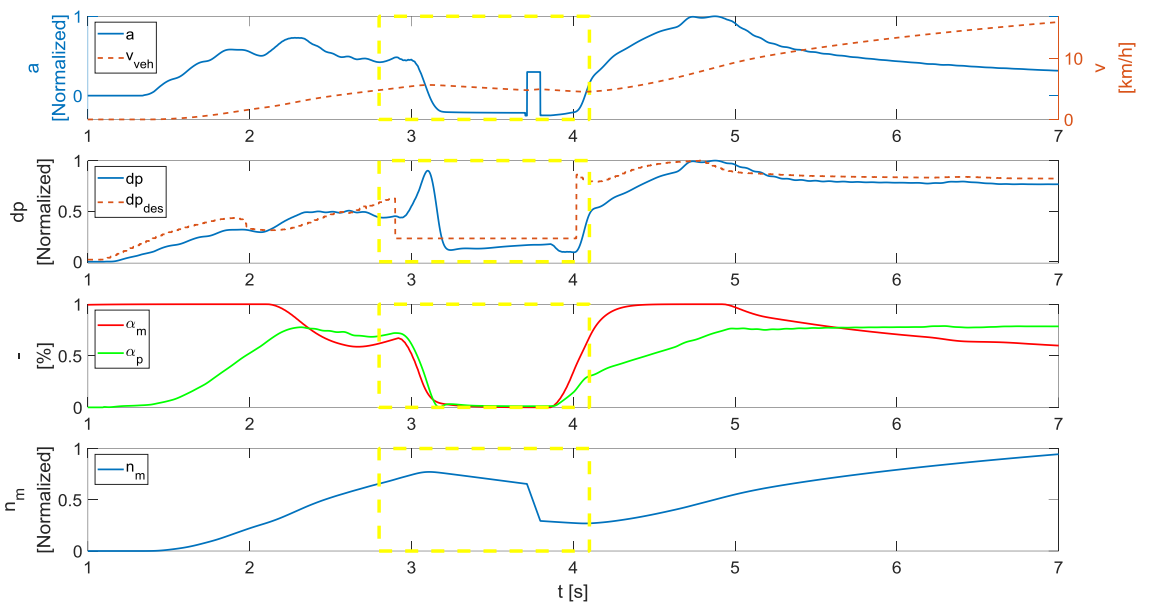

Figure 7: Simulation result of shifting with angle based control algorithm

\section{Simulation result of $A B C A$}

The simulation result in Figure 7 shows that unlike pressure-based control, the pump has no burden within the synchronization phase. Thus, vehicle speed does not affect the shift performance except shift duration. A pressure peak occurs since the motor swivel back to zero position. Correspondingly, the pump also swivels back to an almost zero position. The desired pressure is set to a certain number in order to help the motor swivel faster. Based on the simulation results, we employed the ABCA concept.

\section{SHIFT MANAGER}

A complete shift function shall not only include a mature shift process but also a reasonable shift manager who determines when the machine shall change its gear.

The original purpose of designing hydrostatics with a synchronized gearbox was this: with the first gear, the machine can have higher torque whilst with the second gear, the machine can drive faster than pure hydrostatics. Furthermore, the energy loss can be considerably reduced. However, the additional synchronized gearbox makes the drivetrain no longer a continuous drivetrain. This may especially be critical with an automatic shifting function. If the vehicle shifts at an unwise situation, it may cause an unexpected accident due to the drive force interruption. For instance, if a machine drives uphill at a low speed, the machine may roll down because of the shifting. To avoid these hazardous events, a shift-prevent function and shiftprotection function is designed, see Figure 8. The shift-prevent function rejects the unwise shift trigger based on the sensor signal, while the shiftprotection function aborts the shifting process whenever the situation is no longer suitable for shifting. The difference of both functions is based on the fact that the shifting process needs about one second, and the situation may change within this one second.

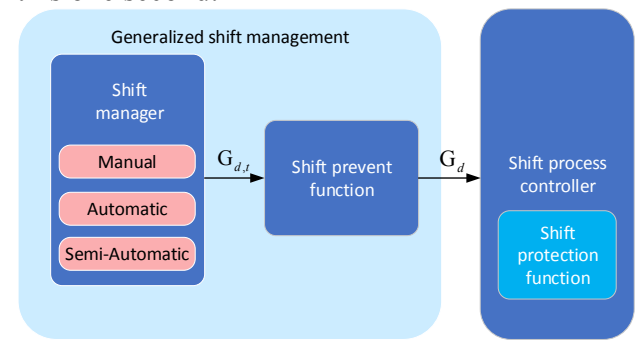

Figure 8: System architecture of shift function

For the novel-drivetrain control system, we suggest an automatic and a semi-automatic shift manager.

\section{Shift manager: Efficiency based}

The energy loss of the hydraulic system can be derived from some basic rules. Generally, swash plate pumps have a higher loss than bent axis motors. The principal factors to calculate loss of 
hydraulic systems are rotation speed, pressure, and swivel angle [17]. From an efficiency perspective, a mechanical gearbox can undoubtedly give the system the potential to improve its efficiency by selecting the gear ratio appropriately. According to e.g. Ivantysyn and Ivantysynova, the loss model of hydraulic components can be mainly illustrated by the following equations [18].

$$
\begin{aligned}
& Q_{V}=\sum_{i=0}^{f_{1}} \sum_{j=0}^{f_{2}} \sum_{k=0}^{f_{3}} K_{Q, k j i} \cdot n^{i} \cdot V_{i}^{j} \cdot \Delta p^{k} \\
& M_{V}=\sum_{i=0}^{f_{1}} \sum_{j=0}^{f_{2}} \sum_{k=0}^{f_{3}} K_{M, k j i} \cdot n^{i} \cdot V_{i}^{j} \cdot \Delta p^{k}
\end{aligned}
$$

Predominantly, the impact factors, such as the rotational speed of hydraulic motor and pressure, have a positive correlation with energy loss. In contrast, the swivel angle has a negative correlation with efficiency. In the first gear, the rotational speed of hydraulic motor and swivel angle of the pump are higher while the pressure inside of closed circuit and swivel angle of motor are lower compared to the second gear if we need to produce the same drive torque at the same vehicle speed. To optimize the efficiency by using appropriate gear selection, we calculate the loss with the different gears in advance and then choose the gear that has been considered as lower energy loss. The basic idea here is that we first measure the loss of hydraulic components and after that, we build an approximation model whose prediction of loss is similar to reality. Based on the estimation of energy loss by approximation model, a gear selector will output the appropriate gear.

\section{Shift manager: Semi-automatic shift strategy}

In contrast to the efficiency-based shifting manager, a semi-automatic shift strategy is recommended. One disadvantage of automatic shift function is the somehow unpredictable shift events leading to an unsafe feeling. With semiautomatic shift strategy, as shown in Figure 9, there are two regions where the shift manager will send the shift request.

Outside of these two regions, for example, at the place where the circle is, the machine will retain its current gear. By applying this strategy, the driver can easily operate between maximum drive torque with second gear or downshift to first gear since there is a large error space between them, providing a much better psychological safety. The dashed line shows the border to achieve a maximum drive torque with the second gear. Beyond this dashed line, for example, at place where pentagon is, the power manager limits the request from the drivetrain solution. Therefore, no additional energy loss occurs.

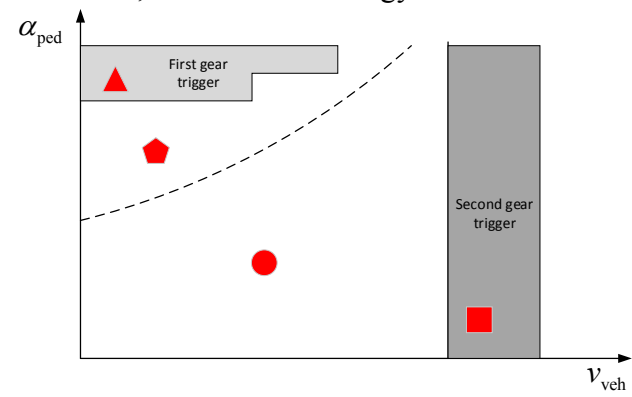

Figure 9: Semi-automatic shift algorithm

\section{VEHICLE MEASUREMENT}

The test machine is a 10t hydrostatic wheel loader with a synchronized gearbox. In this section, we will only experimentally validate the swivelangle-based solution, which is considered as a more feasible solution based on the simulation, on our wheel loader.

Most of the "innovations" shows that they have a better performance than state-of-the-art solution, but they have an advantage only in some instances but lose the total competition compared to mass production. Certainly, a real innovation should always have a better performance, or at least in the most common cases. In this section, we consciously show the test results for the most common use case, shifting at speed of 5 and 14 $\mathrm{km} / \mathrm{h}$. Moreover, intensive tests are running in all use cases to ensure the robustness of the solution.

\subsection{The criteria}

The criteria can be summarized as follows:

- The shift duration must be as short as possible. Since mobile machines usually drive on the site, and the road is unsmooth, an extended drive force interruption may cause a roll down situation, and thus a shorter duration can avoid many problems.

- Usually, the driver triggers a downshift because they need more tractive effort, and the vehicle is at low speed. Therefore, 

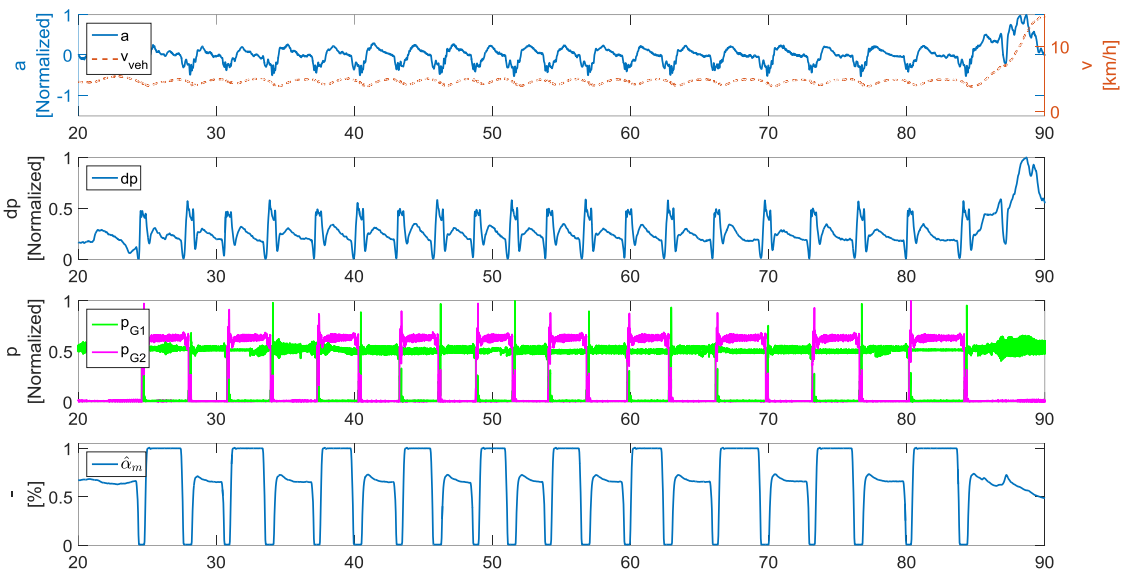

Figure 10: Measurement result of shifting at $5 \mathrm{~km} / \mathrm{h}$

the shift duration of the downshifting process is more critical than the upshift.

- There should be no vehicle jerk due to the shifting process.

- The pressure peak during the shifting process should not be higher than 300 bar, which may harm the pipe lifetime.

\subsection{Results}

As Figure 10 shows, the shifting performance is repeatable, which illustrates the robustness of our control system. The shift duration for upshifting and downshifting process are always about 1 second. The pressure peak is not more than 150 bar during the shifting process, and only a tiny proportion goes to 150 bar. The average pressure is roughly 100 bar. PG1 and PG2 denote the pressure exerts on the operation piston to let the synchronizer to engage with first gear and second gear, correspondingly. For instance, PG1=30bar shows that the first gear is engaged. For these measurements, we simply maintain the drive pedal position. The machine's velocity slightly
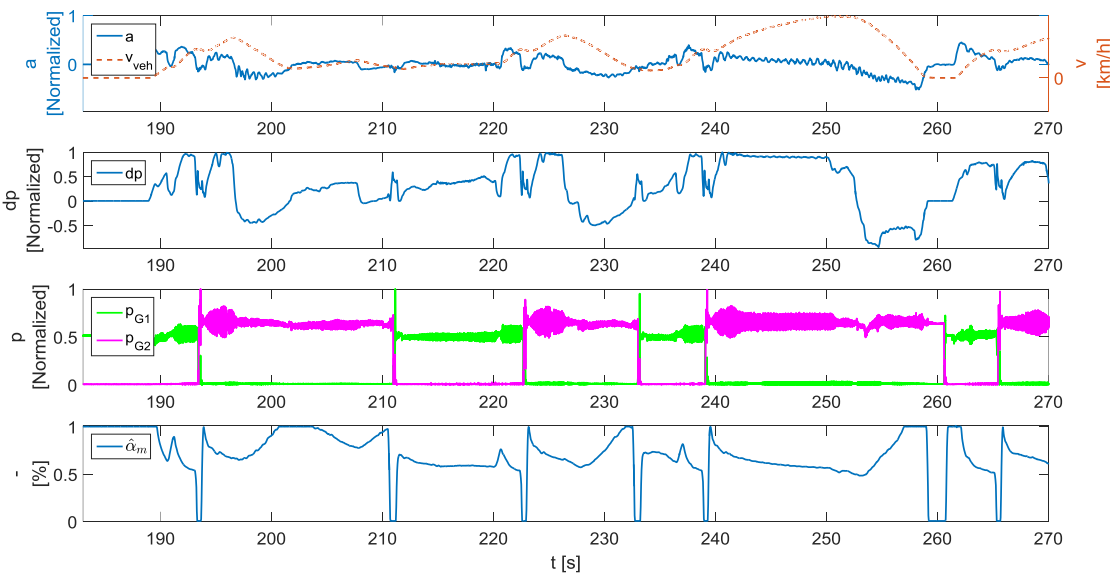

Figure 11: Measurement result of shifting at $14 \mathrm{~km} / \mathrm{h}$ 
fluctuates at about $5 \mathrm{~km} / \mathrm{h}$, indicating that the drive torque on the wheels is almost the same before and after shifting.

From a system efficiency point of view, $14 \mathrm{~km} / \mathrm{h}$ is usually considered as the dividing point where first gear or second gear should be used. Thus, Figure 11 illustrates the shifting process at $14 \mathrm{~km} / \mathrm{h}$. The measurement results show that the most crucial criterion, shift duration, has almost no difference between at 5 and $14 \mathrm{~km} / \mathrm{h}$. At relatively high speed, the speed different between ongoing and upcoming gear is larger than that at a lower speed causing a longer synchronization process whilst a higher pressure inside the closedcircuit accelerates the swivel speed of the hydraulic motor. The interactions compensate each other so that the shift duration in total is almost the same. The difference here is the pressure peak during shifting since the pressure in the closed circuit is already higher before shifting. Notice that at the time $260 \mathrm{~s}$, the shift duration is much longer than the other occurrences because the vehicle is stopped. In standstill, the pressure inside of the closed-circuit is set to zero so that the vehicle will not move in this case. Owing to lack of pressure in the actuator, hydraulic motor swivels back much more slowly, and the operation piston also needs more time to complete the engagement. Nevertheless, nothing can be harmed since the machine has already stopped. Obviously, the velocity and acceleration of the machine are smooth, and no jerk occurs. Notice that, the measurement result shows a better performance than simulation result since we adjust the calibration parameter.

\section{GENERALIZED ARCHITECTURE}

The control architecture proposed in this paper is initially for a synchronized gearbox drivetrain solution. However, mobile machines have a broad spectrum resulting in limited usage of the above-mentioned control algorithm. In order to make our control algorithm more useful, we designed a general controller architecture. With such an architecture, all kinds of commonly used drivetrain solutions can be controlled with the same architecture only with a little effort in adapting the software. Towards this goal, we consciously keep the interface of each module the same. Figure 12 illustrates the basic idea.

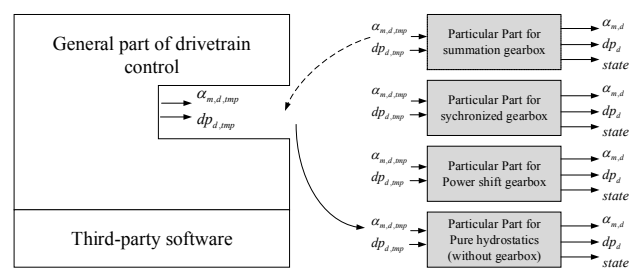

Figure 12: Purpose of the general control architecture

The controller is divided into a general part and particular parts. The particular part is the part that is designed only for a certain kind of gearbox.

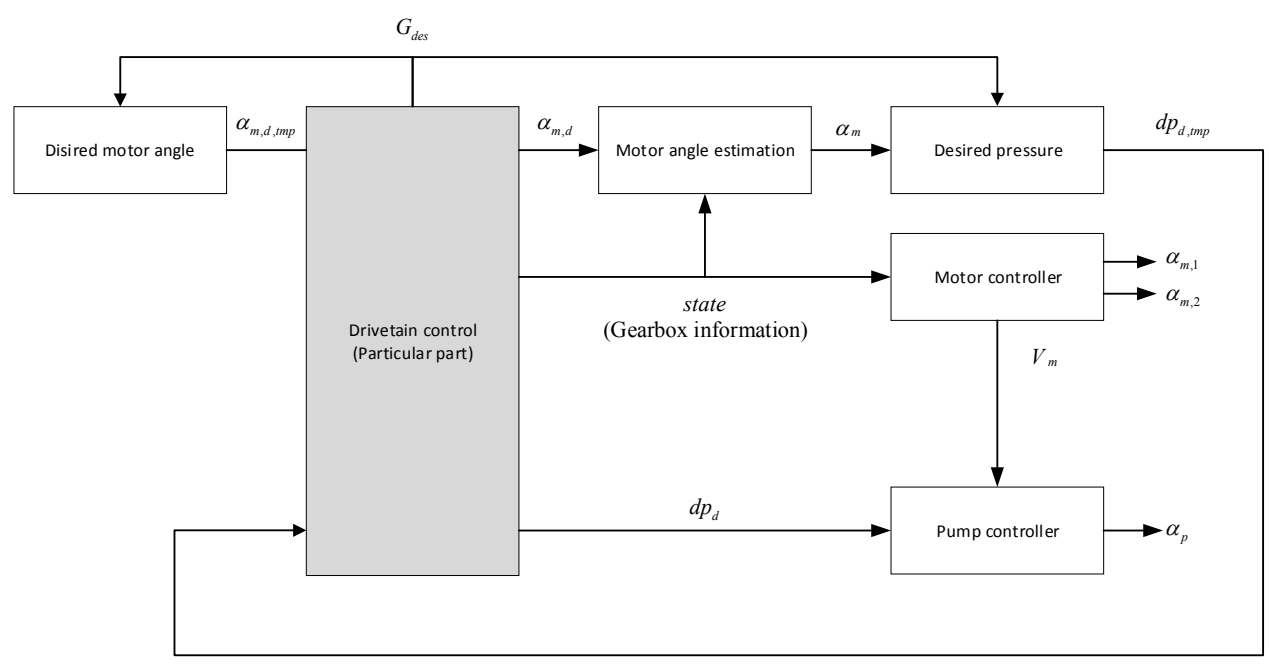

Figure 13: Control scheme with general architecture 
Based on the control signal from general part, the particular part overwrites the control signals considering the requirement of the currently used gearbox. The third-party software can be connected to the general part to fulfill customers' individual requirements.

\section{CONCLUSION AND OUTLOOK}

In this paper, we introduced our novel hydrostatic-mechanical drivetrain solution that adopted the primary torque control concept. Starting with initial simulation studies, the potential shift strategies, such as pressure-based shift strategy and swivel-angle-based shift strategy, were compared and the swivel angle based control algorithm was finally selected. Vehicle tests validated the swivel-angle-based control algorithm approach. As the experimental results show, the novel hydrostatic-mechanical transmission control strategy can efficiently enhance the shift performance of a hydrostatic drivetrain with synchronized gearbox. The main contributions of this novel system can be summarized as follows. Firstly, due to the reduction of calibration parameters to one parameter, the calibration process can be decreased from hours to a few minutes in general. Secondly, a better shift quality in terms of drive comfort and system lifetime is reached thanks to pressure controller. Thirdly, no special experience is needed to achieve the best shift performance of this system, novice drivers can easily operate it. Lastly, a flexible system architecture was drawn so that not only the hydrostatics with a synchronized gearbox but also other commonly used drivetrain solutions, such as with a summation gearbox or with a power shift gearbox, can be controlled under this algorithm.

In addition, the system indicates that it is beneficial to improve the drivetrain efficiency by adopting an appropriate shift manager since it enables hydraulic components to have better working conditions.

\section{OUTLOOK}

Although we can reduce the duration of tractive effort interruption, we cannot eliminate it due to the principle of the synchronizer. A mature drivetrain solution to avoid drive torque interruption is hydrostatics with a summation gearbox. However, we need an additional hydraulic motor for this drivetrain solution and thus increase the system cost. Based on the above analysis, we believe it is a sensible idea to use a dual-clutch gearbox to achieve a continuous drivetrain torque during the shifting process even with only one hydraulic motor is a sensible idea. In the past, power shift hydrostatic drivetrain with a dual clutch transmission had a small market share. Thanks to its high dynamics, we believe primary torque control can strongly minimize the vehicle jerk during the shifting process as the dual clutch transmission is used. As the next step, we are going to extend our hydrostatic-mechanical drivetrain solution to power shift, in this case, dual-clutch gearbox.

Another research goal must be the improvement of the efficiency of our primary control algorithm during $\mathrm{Y}$ cycles. Unlike secondary control, primary torque concept does not have an accumulator to recuperate the kinetic energy of machines. Instead, it needs a competent solution to regenerate the energy.

\section{NOMENCLATURE}

$\begin{array}{ll}V_{p} & \text { Displacement of the pump } \\ V_{m} & \text { Displacement of the motor } \\ \eta_{e} & \text { Mechanical efficiency } \\ n_{p} & \text { Rotation speed of the pump } \\ n_{m} & \text { Rotation speed of the motor } \\ \alpha_{m} & \text { Swivel angle of the motor } \\ \eta_{o f f} & \text { Offset of the swivel angle of the pump } \\ \alpha_{p, d e s} & \text { Desired pump angle } \\ \alpha_{m, d e s} & \text { Desired motor angle } \\ d p_{d} & \text { Desired pressure difference } \\ \alpha_{m, d, t m p} & \text { The temporarily desired motor swivel angle } \\ d p_{d, t m p} & \text { The temporarily desired pressure difference } \\ G_{d e s} & \text { Desired gear } \\ \alpha_{m, 1} & \text { Swivel angle of the temporary motor } \\ \alpha_{m, 2} & \text { Swivel angle of the permanent motor } \\ \alpha_{p} & \text { Actual swivel angle of the pump }\end{array}$

\section{REFERENCES}

[1] Geimer M, Pohlandt C (2014) Grundlagen mobiler Arbeitsmaschinen. Karlsruher Schriftenreihe Fahrzeugsystemtechnik, vol 22. KIT Scientific Publishing, Karlsruhe

[2] Mutschler S, Brix N, Xiang Y (2018) Torque Control for Mobile Machines. In: Murrenhoff $\mathrm{H}$ (ed) 11th International Fluid Power Conference. RWTH Publications, Aachen, pp 186-195 
[3] Mercati S, Panizzolo F, Profumo G (2016) Power Split Hydro-mechanical Variable Transmission (HVT) for Off-highway Application. In: 10th International Fluid Power Conference. Technische Universität Dresden, Dresden, pp 69-80

[4] Legner J, Rebholz W., Morrison R. (2016) ZF cPower - Hydrostatic-Mechanical Powersplit Transmission for Construction and Forest Machinery. In: 10th International Fluid Power Conference. Technische Universität Dresden, Dresden, pp 45-52

[5] Murrenhoff H (1999) Systematic Approach to the Control of Hydrostatic Drives. Proceedings of the Institution of Mechanical Engineers, Part I: Journal of Systems and Control Engineering 5(213): 333-347

[6] Murrenhoff H (2008) Servohydraulik Geregelte Hydraulische Antriebe, 3., neu überarbeitete Auflage. Shaker Verlag, Aachen

[7] Kordak R (1981) Neuartige Antriebskonzeption mit sekundärgeregelten hydrostatischen Maschinen. Ölhydraulik und Pneumatik 5(25): 387-392

[8] Bauer G (2016) Ölhydraulik: Grundlagen, Bauelemente, Anwendungen, 11. Auflage. Springer Fachmedien Wiesbaden, Wiesbaden

[9] Backé W (1994) Verlustarme hydrostatische Antriebe. Grundlagen und Anwendungen. VDI Berichte-Verein Deutscher Ingenieure, Düsseldorf 1132: 147-166

[10] Murrenhoff H, Sgro S, Milos Vukovic (2014) An Overview of Energy Saving Architectures for Mobile Applications. In: Murrenhoff H (ed) 9th International Fluid Power Conference. HP-
Fördervereinigung Fluidtechnik, Aachen, pp 238-249

[11] Vukovic M, Leifeld R, Murrenhoff H (2016) STEAM - A Hydraulic Hybrid Architecture for Excavators. In: 10th International Fluid Power Conference. Technische Universität Dresden, Dresden, pp 151-162

[12] Vukovic M, Sgro S, Murrenhoff H (2013) STEAM - A Mobile Hydraulic System with Engine Integration. In: ASME/BATH 2013 Symposium on Fluid Power and Motion Control, Sarasota, V001T01A005-V001T01A005

[13] Sprengel M, Bleazard T, Haria H et al. (2015) Implementation of a Novel Hydraulic Hybrid Powertrain in a Sports Utility Vehicle. IFACPapersOnLine 15(48): 187-194

[14] Sprengel MW (2015) Influence of Architecture Design on the Performance and Fuel Efficiency of Hydraulic Hybrid Transmissions. Dissertations, Purdue University

[15] Hense H, Riggenmann F, Wille T (2008) Shift On Fly: Automatisierung des Schaltens während der Fahrt von Stillstandsschaltgetrieben. In: 5. Kolloquium Mobilhydraulik, Karlsruhe

[16] Fischer R, Küçükay F, Jürgens G et al. (2015) The Automotive Transmission Book. Powertrain. Springer International Publishing Switzerland, Cham

[17] Kohmäscher T (2008) Modellbildung, Analyse und Auslegung hydrosratischer Antriebsstrangkonzepte. Dissertation, RWTH Aachen

[18] Jaroslav I, Ivantysynova M (2003) Hydrostatic Pumps and Motors: Principles, Design, Performance, Modelling, Analysis, Control and Testing. Tech Books International 